\title{
Ultrasonic imaging of standing trees: factors influencing the decay detection
}

\author{
Luis Espinosa \\ Dept. of Mechanical and \\ Mechatronics Engineering \\ Universidad Nacional de \\ Colombia \\ Bogotá, Colombia \\ lfespinosam@unal.edu.co
}

\author{
Flavio Prieto \\ Dept. of Mechanical and \\ Mechatronics Engineering \\ Universidad Nacional de \\ Colombia \\ Bogotá, Colombia \\ faprietoo@unal.edu.co
}

\author{
Loïc Brancheriau \\ UR BioWooEB \\ CIRAD \\ Montpellier, France \\ loic.brancheriau@cirad.fr
}

\author{
Philippe Lasaygues \\ LMA \\ Aix Marseille Univ, CNRS, \\ Centrale Marseille \\ Marseille, France \\ lasaygues@1ma.cnrs-mrs.fr
}

\begin{abstract}
Ultrasound Computed Tomography is a widely used technique for nondestructive control of materials. One application is the evaluation of the inner state of standing trees in urban areas. The quality of the tomographic image depends on several factors, such as the number of probes used and the image reconstruction algorithm. Here we are interested in evaluating the influence of using a reconstruction algorithm adapted to wood anisotropy, compared to the classic methods using an isotropic model, and the effect of numerically increasing the number of sensors and the time-of-flight measurements using an interpolation method, known as sinogram interpolation. A numerical configuration was tested, simulating the presence of an eccentric defect in a wood section. Reconstructed images were compared before and after the interpolation process, and a thresholding was applied to quantify the decay area estimation. A more detailed defect identification was obtained with the reconstruction algorithm adapted to wood anisotropy and the interpolation of the sensors.
\end{abstract}

Keywords—wood, orthotropy, ultrasound, tomography

\section{INTRODUCTION}

Urban trees are vital in modern cities several, offering benefits at ecological and sanitary levels. To manage this resource, modern tools to evaluate their phytosanitary state are available [1]. To analyse the inner structures of trees without modifying their condition, non-destructive imaging methods have been presented as an alternative to the classic drilling techniques, specially using acoustic signals [2]. These acoustic devices create a $2 \mathrm{D}$ image (or $3 \mathrm{D}$ via interpolation) of the cross-section of standing trees using the waves transit times (TOF: time-of-flight) measurements. They have proved to be valuable tools for decay detection [3-6]. Academic approaches using ultrasound signals have been proposed to improve the image resolution [7-9]. However, several issues must be addressed concerning the image quality to improve the diagnostic.

Several factors influence the quality of the acoustic and ultrasonic images [10]. The number of sensors, for example, is directly related to the spatial resolution of the image; a larger number of sensors allows a smaller defective area detection $[11,12]$. The excitation signal used for testing is directly related to the precision of the TOF estimation, affecting the image reconstruction method [13,14]. This image reconstruction method must be adapted to the wood anisotropy condition, in order to avoid the bias introduced by the isotropic hypothesis considering straight-line trajectories $[15,16]$. For wood, the ray paths between the ultrasonic transmitter and the receivers are not straight as for isotropic media; therefore, the image reconstruction method should be adapted to deal with curved rays. The defect position is other factor influencing the detection, with eccentric defects being more difficult to locate [16]. Other factor is related to the definition of the tree geometry, considering that most of the cases the cross-section will differ from a perfect circle, and these variations will modify the image construction.

In this study, we are interested on numerically evaluating the influence of two factors in the reconstruction of ultrasonic tomographic images for standing tree decay detection: the image reconstruction method and the interpolation of the TOF measurements to simulate a higher number of sensors. First, considering the orthotropic condition of wood, TOF numerical estimations for a cross-section of wood were obtained using a raytracing approach [17], a method used mainly in the field of exploration seismography to simulate wave fronts in elastic media. Images were thus obtained using two reconstruction methods: one adapted to curved rays (anisotropy) and other considering straight-line trajectories (isotropic). After, the TOF measurements were interpolated and the images were computed again. A thresholding process was applied to evaluate decay detection performance. Section II presents the numerical model used to obtain the TOF measurements, the interpolation method and the image reconstruction approaches. Section III describes the results obtained for both reconstruction methods, before and after using the interpolation.

\section{Methodology}

\section{A. Forward problem and sinogram interpolation}

TOF numerical measurements (input data for the image reconstruction) were obtained for a wood cross-section, using a raytracing approach [17]. For the simulation, the disk diameter was set to $30 \mathrm{~cm}$, with an eccentric hole with a diameter of 10 $\mathrm{cm}$, located halfway between the disk center and the bark (Fig. 1). 16 sensors were located around the trunk perimeter. Wood mechanical parameters for the model were obtained from 


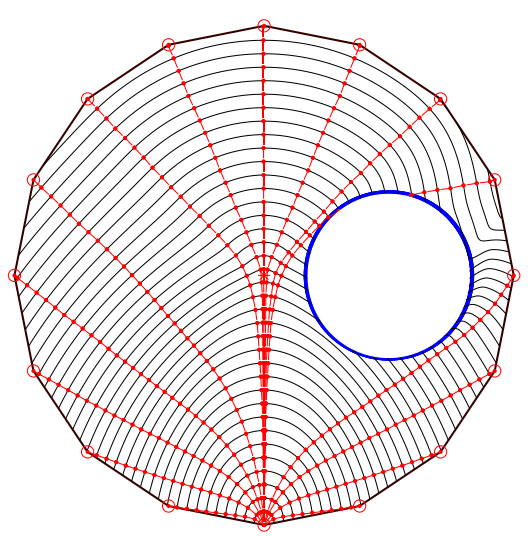

Fig. 1. Forward model used to obtain the TOF estimations using a raytracing approach. Curved rays (in red) were obtained due to wood anisotropy. In black: wavefronts.

published data [18], for Oak species, with $E_{R}=1180 \mathrm{MPa}$ and $E_{T}=614 \mathrm{MPa}$ as the Young's modulus in the radial and tangential directions respectively, $G_{R T}=319 \mathrm{MPa}$ as the shear modulus, $v_{R T}=0.56$ as the Poisson's ratio and $\rho=570 \mathrm{~kg} / \mathrm{m}^{3}$ as the density. The obtained TOF measurements were represented as a sinogram, depicting the TOF values for every pair emitterreceiver in a grid (Fig. 2).

To virtually increase the number of sensors and their corresponding TOF measurements, a linear interpolation of the sensors positions was done to create a virtual sensor between every two real sensors. Considering that the transit time corresponds to a physical measurement, we can suppose that the TOF value between two adjacent sensors could be approximated by their mean value. In this case, the number of sensors was duplicated, so passing from 16 to 32 sensors. Similarly, the TOF value for this new sensor was interpolated from the values of the two surrounding sensors. The resulting image after applying the sinogram interpolation will be two times larger and higher than the image obtained with the

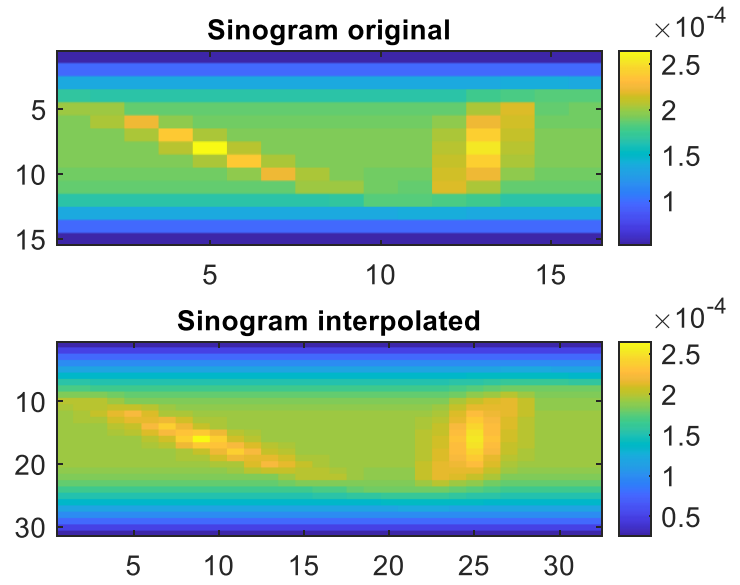

Fig. 2. TOF measurements representation as a sinogram, before (up) and after (bottom) the sinogram interpolation. original TOF measurements.

\section{B. Image reconstruction}

To reconstruct the tomographic images, two methods were considered. First, the typical approach using straight lines trajectories (isotropic material) was done by using the filtered back-projection (FBP) method [19]. Second, we proposed a reconstruction method adapted to the wood anisotropy condition, that iteratively obtains the curved rays to reduce the difference between the input TOF and values obtained via the forward model [17]. This method allowed us to obtain a parametric image that represents the inner mechanical values, in this case the comparison was made using a radial Young modulus $\left(E_{R}\right)$ image, assuming that $\rho$ is known.

To perform an estimation of the decay detection, the images were segmented using a threshold. The threshold values were fixed using the minimum and maximum values in the image, as the minimum value plus a $20 \%, 30 \%$ and a $40 \%$ of the difference between the maximum and the minimum value. To evaluate the classification, the true positive rate (sensitivity) and the false positive rate (fall-out) were computed. The first corresponds to the ratio of correctly identified pixels inside the defective area. The second is the ratio of incorrectly identified pixels, i.e. the pixels classified as defect that are outside the defective area.

\section{RESULTS}

Images were obtained using the TOF estimation before the sinogram interpolation, as presented in Fig. 3 and Fig. 4 for the FBP method and the method considering wood anisotropy respectively. The output image size was increased using a linear interpolation to obtain the same size of the reconstruction after the sinogram interpolation (twice larger and higher).

As expected, using the method that considers the wood anisotropy condition resulted in a more contrasted defective area compared to the straight-line reconstruction method. The

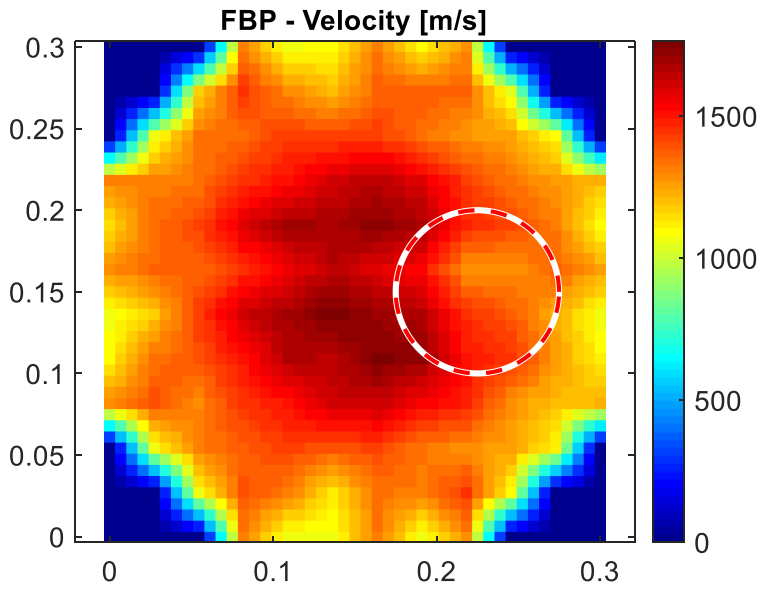

Fig. 3. Reconstructed image using the FBP method with the TOF measurements before the sinogram interpolation. Circle shows the defective area. 


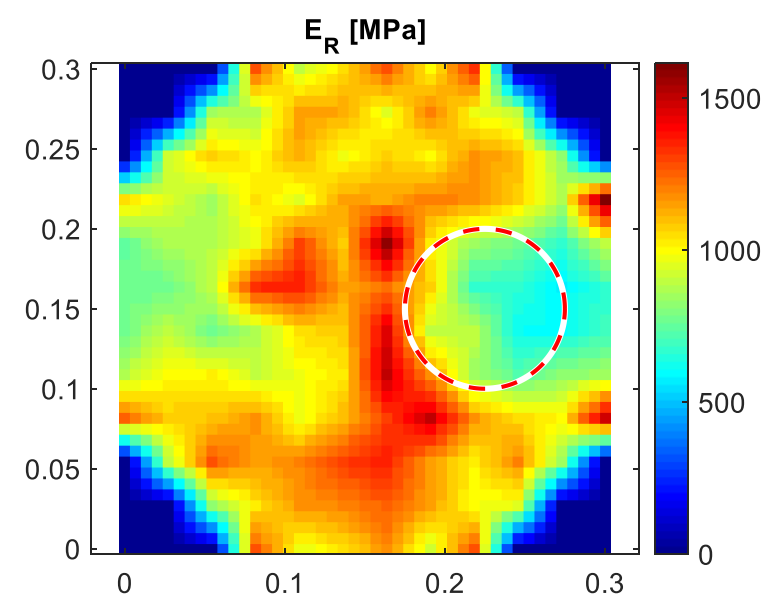

Fig. 4. Reconstructed image using the proposed method considering wood anisotropy with the TOF measurements before the sinogram interpolation. Circle shows the defective area.

bias introduced by the FBP method resulted in a more complex defect identification, as can be observed in Fig. 5, where the threshold was applied. The error introduced by considering straight-line trajectories resulted in lower velocity values in the image borders, so the defect was mixed up with those regions, making difficult the detection. The proposed method considering wood anisotropy resulted in a detection of the defect closer to the actual shape, even if the location was slightly shifted to the border. Also, it is noticeable the apparition of a misclassified region in the opposite side of the defect, when the threshold value increased, mostly due to the approximations done by the method. For the threshold value of $40 \%$ (best case), true positive rate (sensitivity) using the FBP method was $36 \%$, while using the ER image this ratio was $64 \%$. For the FBP method, the false positive rate (fall-out) was $56 \%$, while for the ER image was as lower as $14 \%$.

After the sinogram interpolation, images were computed
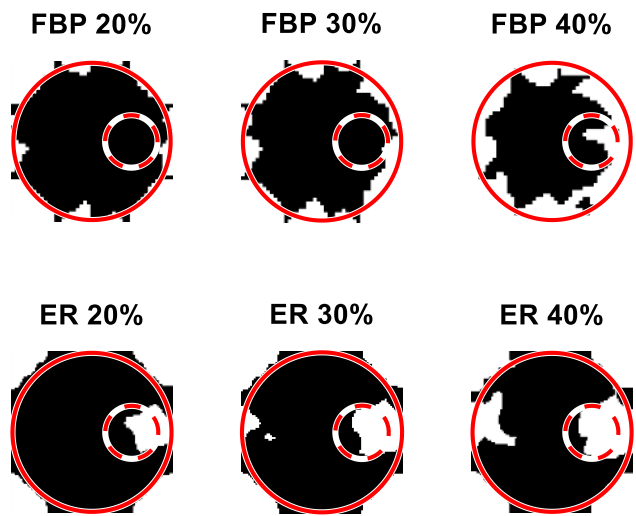

Fig. 5. Segmented images for different threshold levels for the FBP images (up) and the images reconstructed considering wood anisotropy (bottom). White areas represent the estimated defective area. Circle shows the real defective area. again using the two methods, as presented in Fig. 6 and Fig. 7 for the FBP method and the method considering wood anisotropy respectively. For these images, more details appeared, compared to the smoothed images obtained with an interpolation directly applied to the output image in Fig. 3 and Fig. 4. Again, a larger contrast between the defect area and the healthy area was obtained with the proposed reconstruction method, while for the FBP method the gradient of velocities was still making difficult the defect identification.

The thresholding was applied to these two images, as presented in Fig. 8. In the case of the FBP method, for the threshold value of $40 \%$ (best case), the true positive rate increased to $52 \%$ compared to the case before the sinogram interpolation; however, the false positive rate increased too, to a value of $65 \%$. Again, the gradient effect using the straightline reconstruction method interfered with the defective area identification. For the image obtained using the proposed

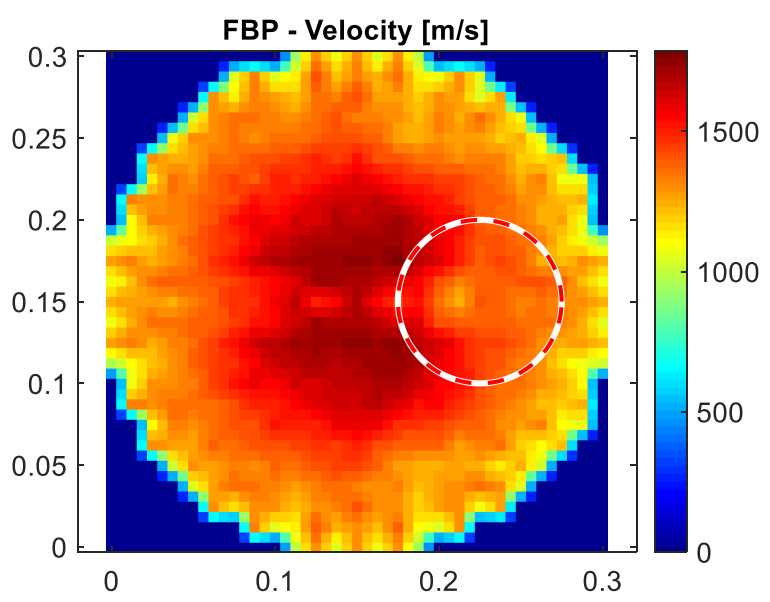

Fig. 6. Reconstructed image using the FBP method with the TOF measurements after the sinogram interpolation. Circle shows the defective area.

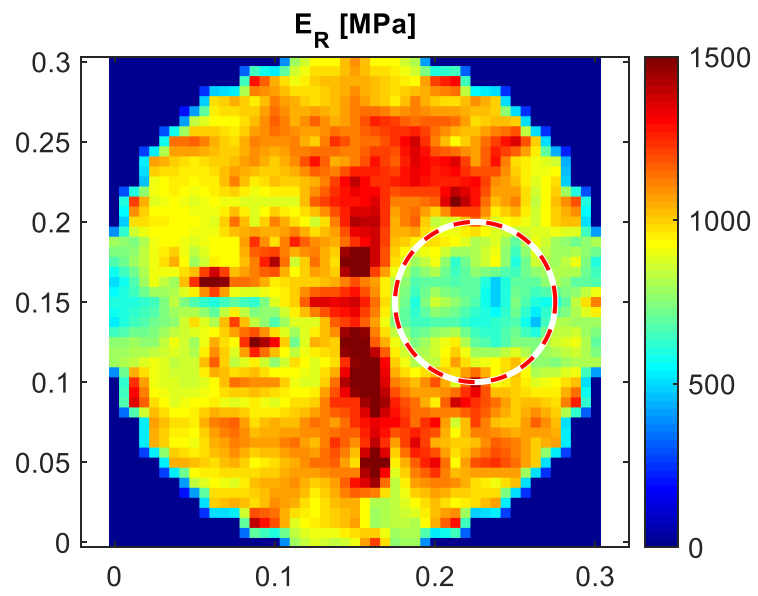

Fig. 7. Reconstructed image using the proposed method considering wood anisotropy with the TOF measurements before the sinogram interpolation. Circle shows the defective area. 
method considering wood anisotropy, the true positive rate increased to $71 \%$ compared to the case before the sinogram interpolation, and the false positive rate was reduced to $12 \%$.

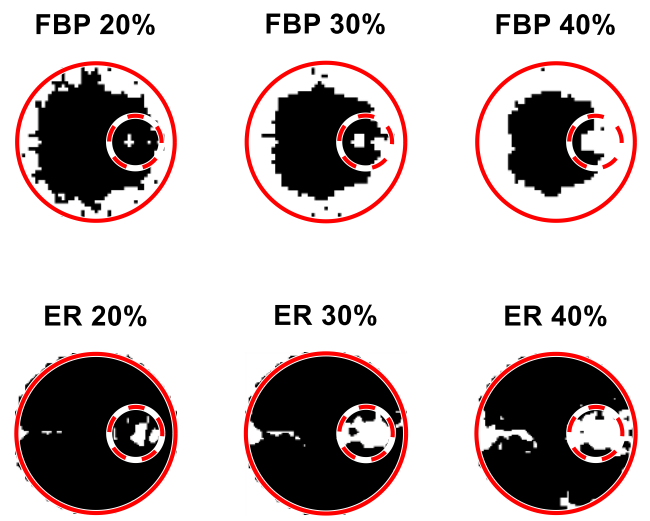

Fig. 8. Segmented images after the sinogram interpolation for different threshold levels for the FBP images (up) and the images reconstructed considering wood anisotropy (bottom). White areas represent the estimated defective area. Circle shows the real defective area.

The number of sensors is directly related to the minimum detectable defect size. The relation between the number of sensors $N$ and the maximum number of pixel that can be reconstructed $M$ is:

$$
M=N^{*}(N-1) / 2
$$

For a configuration with 16 sensors, the size of a squared pixel is limited to $2.5 \mathrm{~cm}$ of length, then smaller defects will not be detected. Increasing to 32 sensors resulted in half the size, i.e. $1.25 \mathrm{~cm}$ for every pixel. Then, other factors are also associated to the minimum detectable size, such as the image reconstruction method, as observed before, and the ultrasonic excitation frequency.

\section{CONCLUSIONS}

Using time-of-flight estimations from a numerical model, tomography images were obtained for a wood cross-section with an eccentric defect. When using a reconstruction algorithm intended for straight ray tomography, the images presented velocity variations from the perimeter to the center that made difficult the discrimination of the inner defect. Considering curved rays due to the anisotropic condition in wood resulted in reconstructed images that allowed a more precise identification of the decay. Applying an interpolation of the sinogram to simulate virtual sensors and to increase the number of time-of-flight measurements resulted in images with more details, which leads to a more accurate defect segmentation.

\section{ACKNOWLEDGMENT}

This work has been carried out in the framework of a project (C16A01) funded by the ECOS Nord program and is a part of Luis Espinosa's Ph.D. thesis (grant from COLCIENCIAS - Departamento Administrativo de Ciencia, Tecnología e Innovación, Colombia). The authors thank the financing of the Universidad Nacional de Colombia (Convocatoria de Apoyo a Tesis de Posgrado, Proyecto Código 40801).

\section{REFERENCES}

[1] J. D. Pokorny, Urban tree risk management: A community guide to program design and implementation. USDA Forest Service, 2003.

[2] R. F. Pellerin and R. J. Ross, Nondestructive evaluation of wood. Madison, Wis. : Forest Products Society, 2002.

[3] E. A. Gilbert and E. T. Smiley, "Picus Sonic tomography for the quantification of decay in white oak (Quercus alba) and hickory (Carya spp.)," Journal of arboriculture, 2004.

[4] D. Johnstone, G. Moore, M. Tausz, and M. Nicolas, "The measurement of wood decay in landscape trees," Arboriculture \& Urban Forestry, vol. 36, no. 3, pp. 121-127, 2010.

[5] C. Rabe, D. Ferner, S. Fink, and F. W. M. R. Schwarze, "Detection of decay in trees with stress waves and interpretation of acoustic tomograms," Arboricultural Journal, vol. 28, no. 1-2, pp. 3-19, Dec. 2004.

[6] X. Wang, Acoustic tomography for decay detection in red oak trees. Madison, WI: USDA, Forest Service, Forest Products Laboratory, 2007.

[7] L. Brancheriau, P. Lasaygues, E. Debieu, and J. P. Lefebvre, "Ultrasonic tomography of green wood using a non-parametric imaging algorithm with reflected waves," Ann. For. Sci., vol. 65, no. 7, pp. 712-712, Jan. 2008.

[8] C.-J. Lin et al., "Application of an ultrasonic tomographic technique for detecting defects in standing trees," International Biodeterioration \& Biodegradation, vol. 62, no. 4, pp. 434-441, Dec. 2008.

[9] R. Martinis, L. V. Socco, L. Sambuelli, G. Nicolotti, O. Schmitt, and V. Bucur, "Tomographie ultrasonore pour les arbres sur pied," Annals of Forest Science, vol. 61, no. 2, pp. 157-162, Mar. 2004.

[10] A. Arciniegas, F. Prieto, L. Brancheriau, and P. Lasaygues, "Literature review of acoustic and ultrasonic tomography in standing trees," Trees, vol. 28, no. 6, pp. 1559-1567, 2014.

[11] L. Wang, H. Xu, C. Zhou, L. Li, and X. Yang, "Effect of sensor quantity on measurement accuracy of log inner defects by using stress wave," Journal of Forestry Research, vol. 18, no. 3, pp. 221-225, Sep. 2007.

[12] A. Arciniegas, L. Brancheriau, P. Gallet, and P. Lasaygues, "TravelTime Ultrasonic Computed Tomography Applied to Quantitative 2-D Imaging of Standing Trees: A Comparative Numerical Modeling Study," Acta Acustica united with Acustica, vol. 100, no. 6, pp. 10131023, Nov. 2014.

[13] A. Arciniegas, L. Brancheriau, and P. Lasaygues, "Tomography in standing trees: revisiting the determination of acoustic wave velocity," Annals of Forest Science, vol. 72, no. 6, pp. 685-691, Sep. 2015.

[14] L. Espinosa, J. Bacca, F. Prieto, P. Lasaygues, and L. Brancheriau, "Accuracy on the Time-of-Flight Estimation for Ultrasonic Waves Applied to Non-Destructive Evaluation of Standing Trees: A Comparative Experimental Study," Acta Acustica united with Acustica, vol. 104, no. 3, pp. 429-439, May 2018.

[15] H. Maurer et al., "A simple anisotropy correction procedure for acoustic wood tomography," Holzforschung, vol. 60, no. 5, pp. 567-573, Jan. 2006.

[16] L. Espinosa, F. Prieto, and L. Brancheriau, "Ultrasonic imaging for nondestructive evaluation of standing trees: effect of anisotropy on image reconstruction," in Thirteenth International Conference on Quality Control by Artificial Vision 2017, 2017, vol. 10338, p. 1033808. 
[17] L. Espinosa, F. Prieto, L. Brancheriau, and P. Lasaygues, "Effect of wood anisotropy in ultrasonic wave propagation: A ray-tracing approach," Ultrasonics, vol. 91, pp. 242-251, Jan. 2019.
[18] D. Guitard, Mécanique du matériau bois et composites. Cepadues Editions, 1987.

[19] A. Kak and M. Slaney, Principles of Computerized Tomographic Imaging. Society for Industrial and Applied Mathematics, 2001. 